\title{
Comparison of the Gamma-Ray Burst Sensitivity of Different Detectors
}

\author{
David L. Band ${ }^{1}$ \\ GLAST SSC, Code 661, NASA/Goddard Space Flight Center, Greenbelt, MD 20771 \\ dband@lheapop.gsfc.nasa.gov
}

\begin{abstract}
Gamma-ray burst detectors are sensitive at different energies, complicating the comparison of the burst populations that they detect. The instrument teams often report their detector sensitivities in their instruments' energy band. I propose that sensitivities be reported as the threshold peak photon flux $F_{T}$ over the 1-1000 keV energy band for a specific spectral shape. The primary spectral parameter is $E_{p}$, the energy of the maximum $E^{2} N_{E} \propto \nu f_{\nu}$. Thus $F_{T}$ vs. $E_{P}$ is a useful description of a detector's sensitivity. I find that Swift will be marginally more sensitive than BATSE for $E_{p}>100 \mathrm{keV}$, but significantly more sensitive for $E_{p}<100 \mathrm{keV}$. Because of its small field-of-view and low energy sensitivity, the FREGATE on HETE-2 is surprisingly sensitive. Both the WFC on BeppoSAX and the WXM on HETE-2 are/were sensitive for low $E_{p}$. As expected, the GBM on GLAST will be less sensitive than BATSE, while EXIST will be significantly more sensitive than Swift. The BeppoSAX GRBM was less sensitive that the WFC, particularly at low $E_{p}$.
\end{abstract}

Subject headings: gamma-rays: bursts

\section{Introduction}

The gamma-ray burst missions that have flown in the past 15 years have reported that bursts are hard (e.g., BATSE-Preece et al. 2000; Mallozzi et al. 1995) or soft (e.g., GingaStrohmayer et al. 1998). However these missions had different sensitivities to hard and soft bursts, and have reported the threshold burst intensities using a variety of different measures,

\footnotetext{
${ }^{1}$ Joint Center for Astrophysics, Physics Department, University of Maryland Baltimore County, 1000 Hilltop Circle, Baltimore, MD 21250
} 
such as peak flux or energy fluence measured over different energy bands. To synthesize the results of these mission (and determine whether they are mutually consistent), and to compare the capabilities of past, current, and proposed missions, we need common measures of burst intensity and hardness, and we need to express a burst detector's capabilities in terms of those common measures.

I propose to characterize bursts by the peak photon flux integrated over $1-1000 \mathrm{keV}$ averaged over one second, and by the spectrum's peak energy $E_{p}$ during this one second. The peak energy $E_{p}$ is the energy of the maximum of $N(E) E^{2}$ (proportional to $\nu F_{\nu}$ )-the energy flux per logarithmic energy (frequency) band; $E_{p}$ is a first order measure of the spectral hardness. The choice of peak photon flux (as opposed to energy fluence, energy flux, or total photon fluence) is based not on the physics of bursts (i.e., theories of fundamental burst properties) but on detector triggers: most detectors trigger on a statistically significant increase in the count rate over a specified energy band. The detection threshold is the minimum peak count rate for which the detector would have triggered. Even though detectors trigger on the count rate in different energy bands, the peak count rate can be translated into the peak photon flux over a fiducial energy band with knowledge of the burst spectrum and the detector's energy response. Note that the count rate is a detector-dependent quantity while the photon flux is an intrinsic description of the burst photons arriving in the Solar System. Therefore, the peak photon flux over a common energy band provides a convenient operational measure of burst intensity.

Because few bursts have lightcurves where the maximum flux is constant over seconds, the peak photon flux will depend on the accumulation time $\Delta t$, the time resolution with which the flux is measured. Although most detectors trigger on a variety of accumulation times, $\Delta t=1 \mathrm{~s}$ is usually included in a detector's set of accumulation times. In addition, a detector's sensitivity for one value of $\Delta t$ can be translated into the average sensitivity for other values using an ensemble of burst lightcurves (Band 2002). Therefore I use $\Delta t=1 \mathrm{~s}$ for this work.

Many studies have presented results using peak photon fluxes in the $50-300 \mathrm{keV}$ band, principally because this was main trigger band of the Burst and Transient Source Experiment (BATSE) on the Compton Gamma-Ray Observatory (CGRO). While any fiducial energy band can be used, particularly soft transient events, such as the recently-discovered X-Ray Flashes (XRFs, which may or may not be related to classical gamma-ray bursts-Heise et al. 2001), will produce little flux in the $50-300 \mathrm{keV}$ band. Therefore I choose to use the 1-1000 keV band because most burst detectors operate within this broad band, and most bursts have $E_{p}$ in this band.

This study focuses predominantly on a comparison of the sensitivity of a number of 


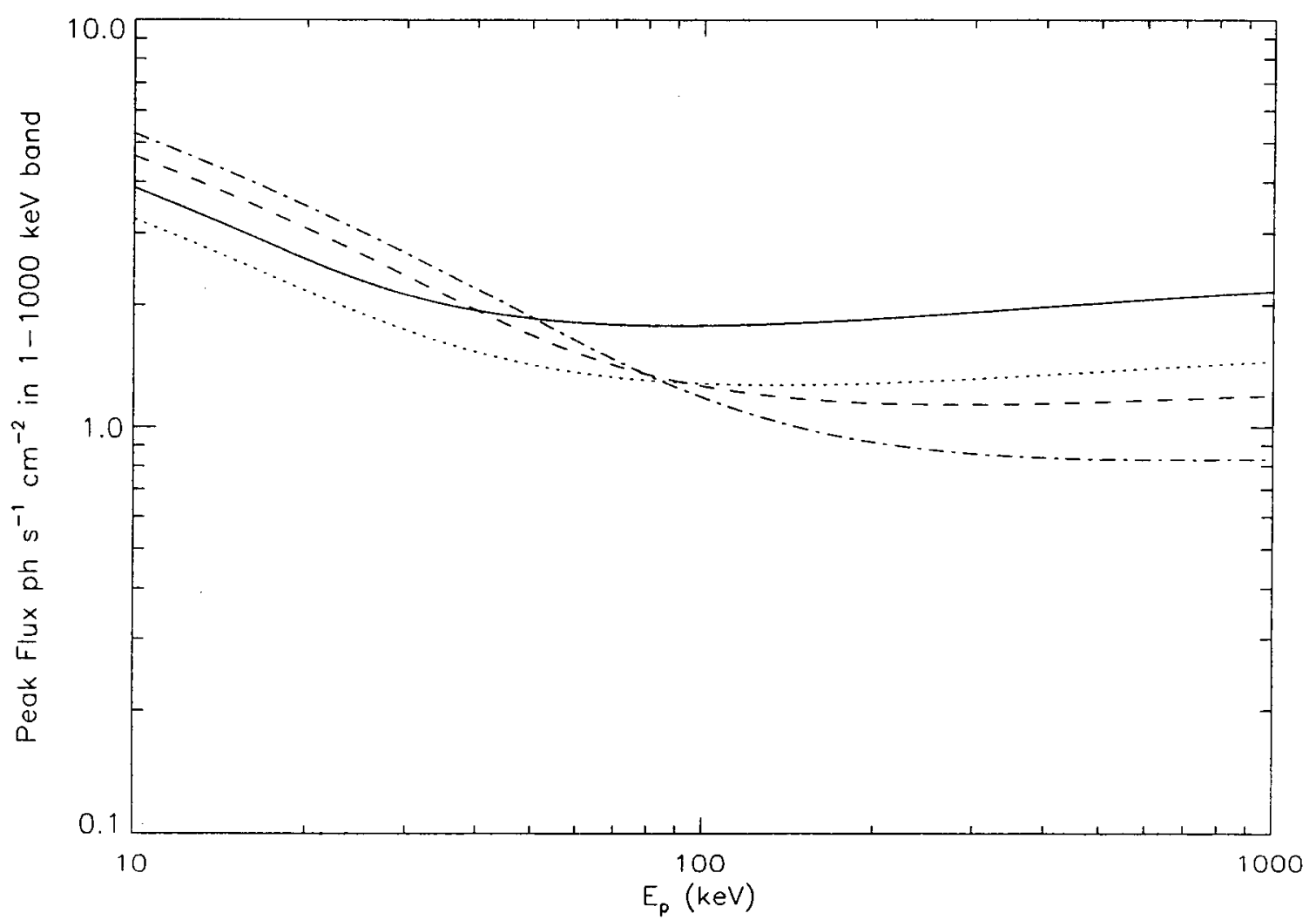

Fig. 10.- Peak flux (1-1000 keV) threshold for the different Swift energy trigger bands. The GRB model with $\alpha=-1$ and $\beta=-2$ is assumed. The solid line is for the $15-30 \mathrm{keV}$ band, dotted for $15-50 \mathrm{keV}$, dashed for $30-75 \mathrm{keV}$ and dot-dashed for $50-150 \mathrm{keV}$. 


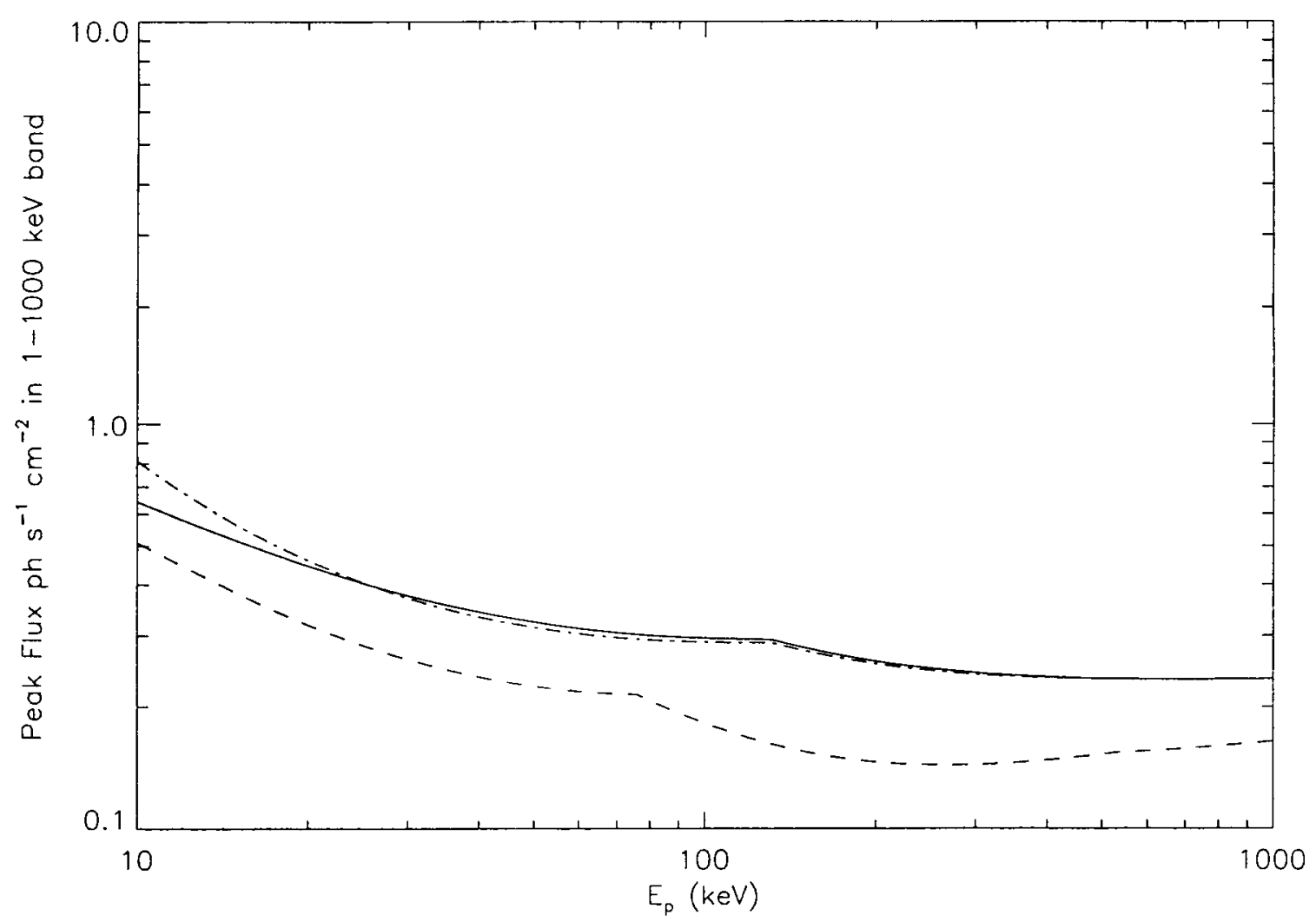

Fig. 9.- Peak flux (1-1000 keV) threshold of one EXIST telescope. Solid line- $\alpha=-1$, $\beta=-2$; dashed line $-\alpha=-0.5, \beta=-2$; dot-dashed line $-\alpha=-1, \beta=-3$. 


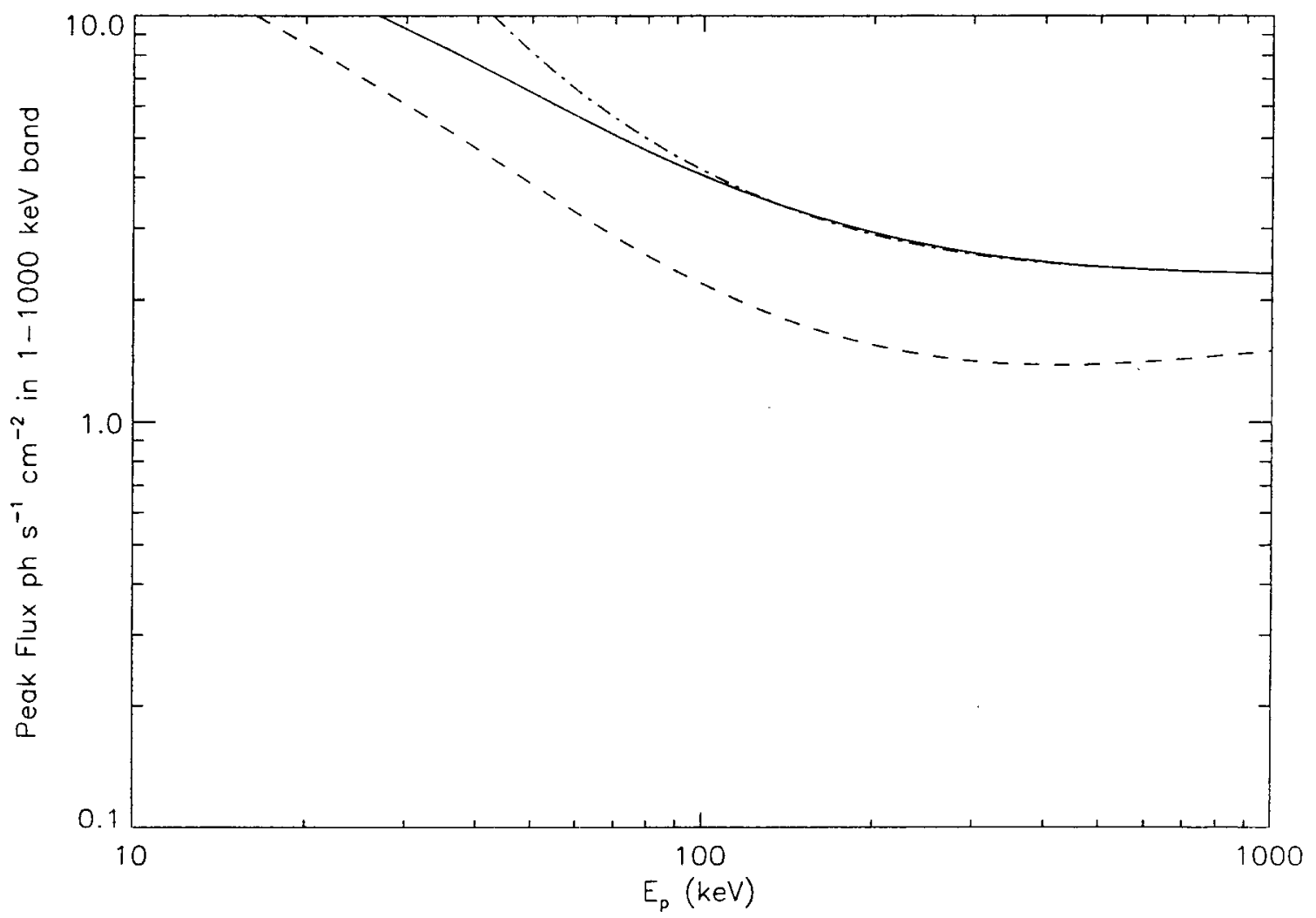

Fig. 8. - Peak flux (1-1000 keV) threshold of GBM NaI detectors. Solid line- $\alpha=-1$, $\beta=-2$; dashed line $\alpha=-0.5, \beta=-2$; dot-dashed line $\alpha=-1, \beta=-3$. 


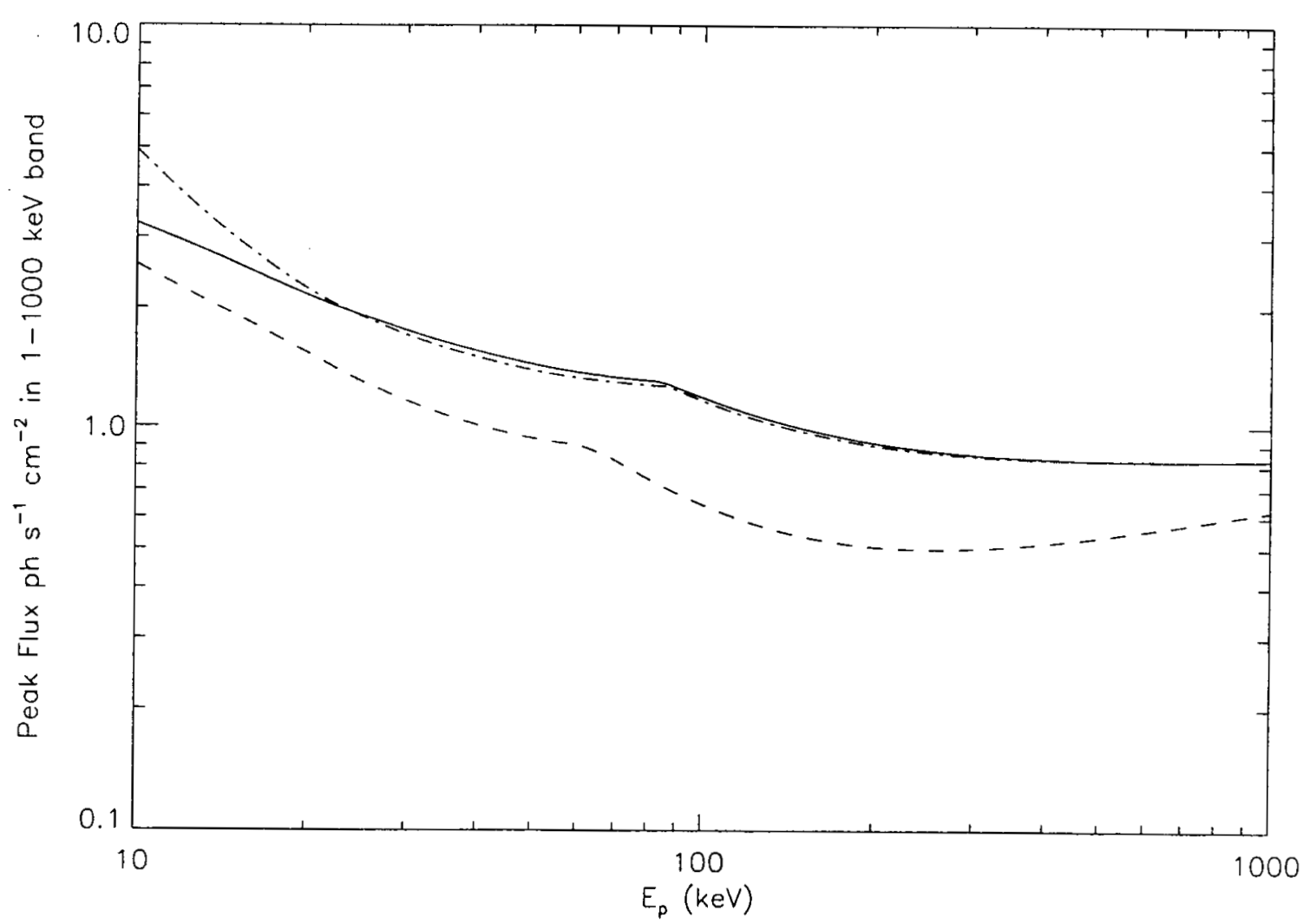

Fig. 7. - Peak flux (1-1000 keV) threshold of the Swift BAT detector. Solid line $-\alpha=-1$, $\beta=-2$; dashed line $\alpha=-0.5, \beta=-2$; dot-dashed line $-\alpha=-1, \beta=-3$. 


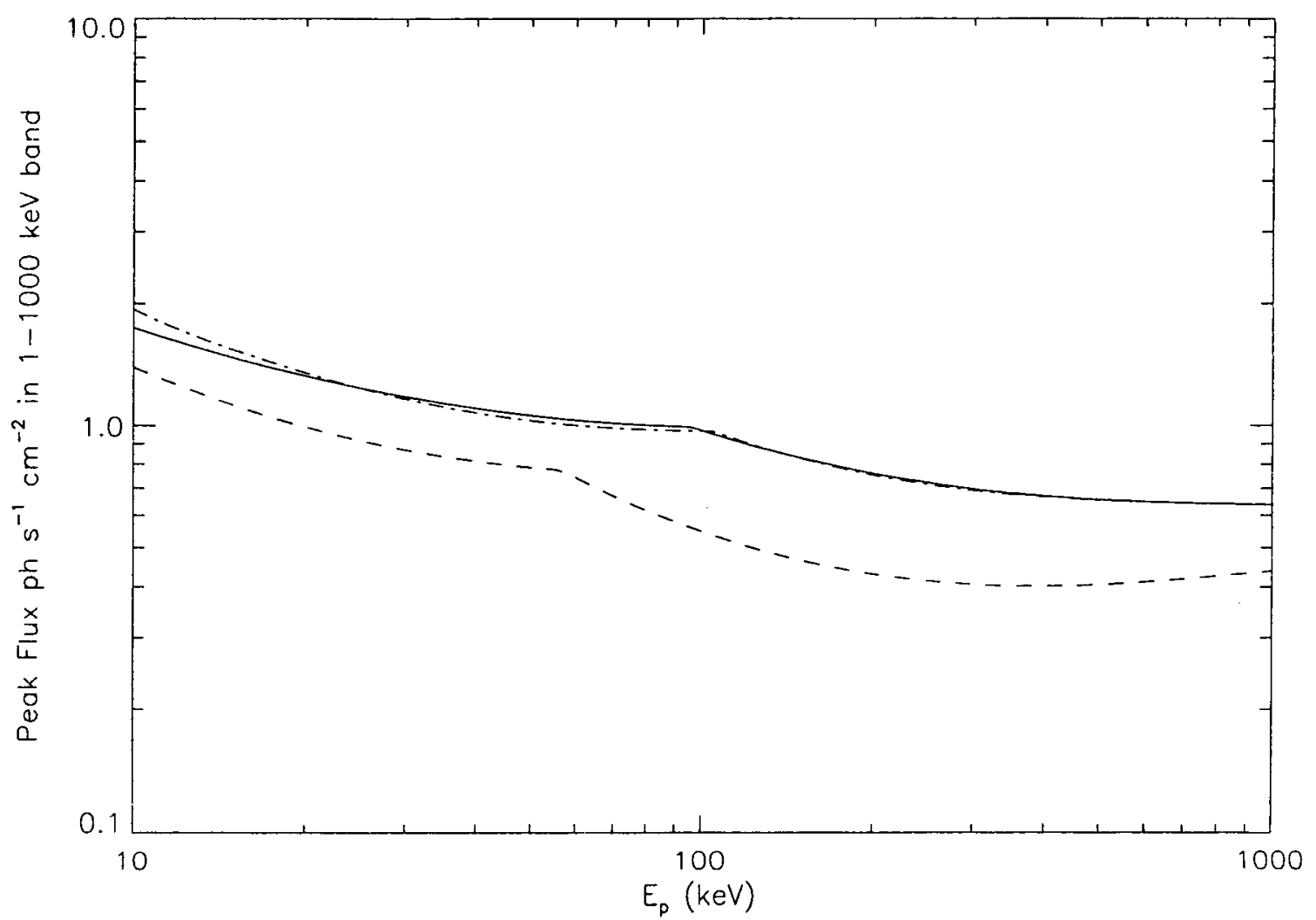

Fig. 6. - Peak flux (1-1000 keV) threshold of the FREGATE detectors. Solid line $-\alpha=-1$, $\beta=-2$; dashed line $-\alpha=-0.5, \beta=-2$; dot-dashed line $\alpha=-1, \beta=-3$. 


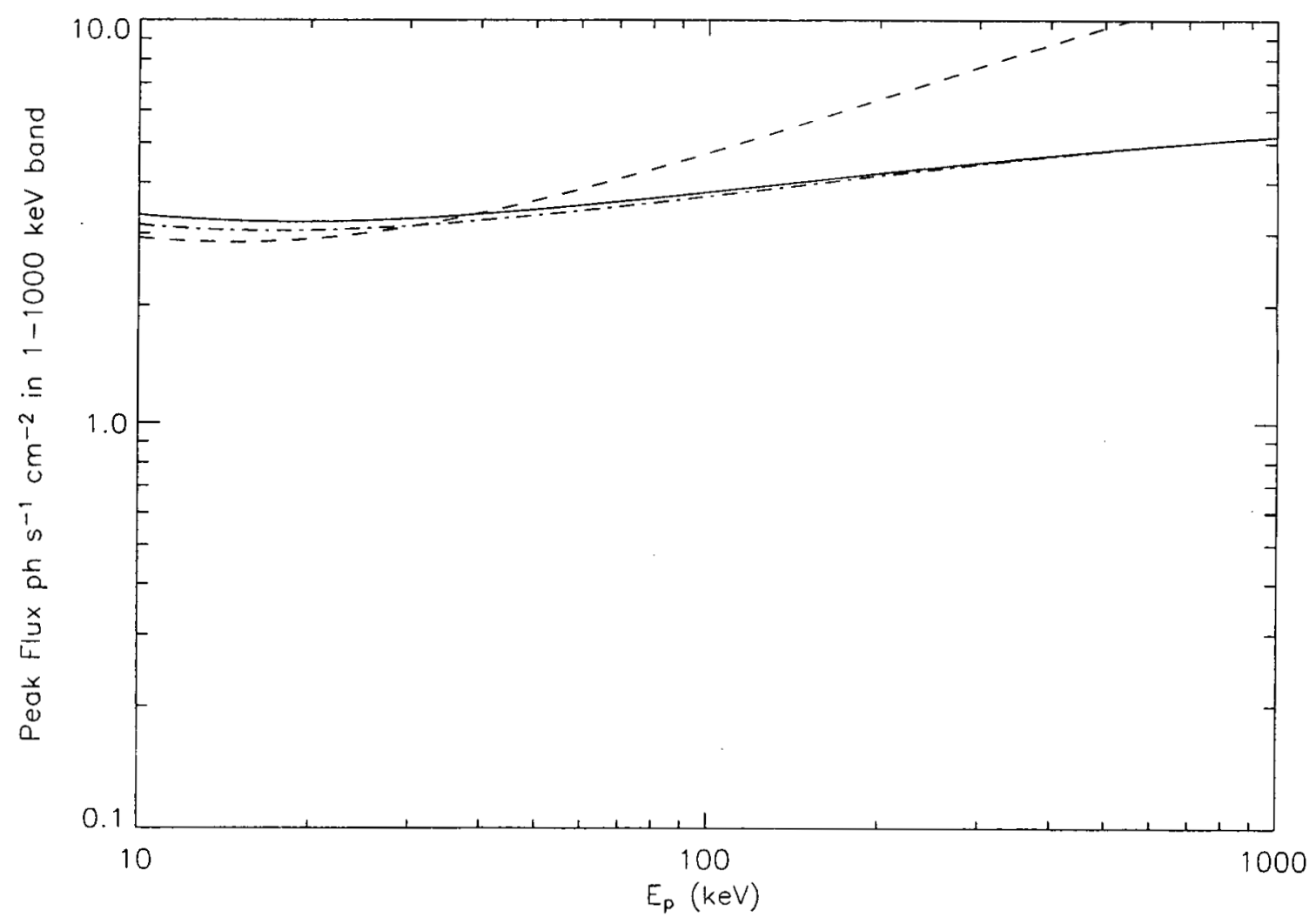

Fig. 5.- Peak flux (1-1000 keV) threshold of the WXM detectors. Solid line $\alpha=-1$, $\beta=-2$; dashed line $-\alpha=-0.5, \beta=-2$; dot-dashed line- $\alpha=-1, \beta=-3$. 


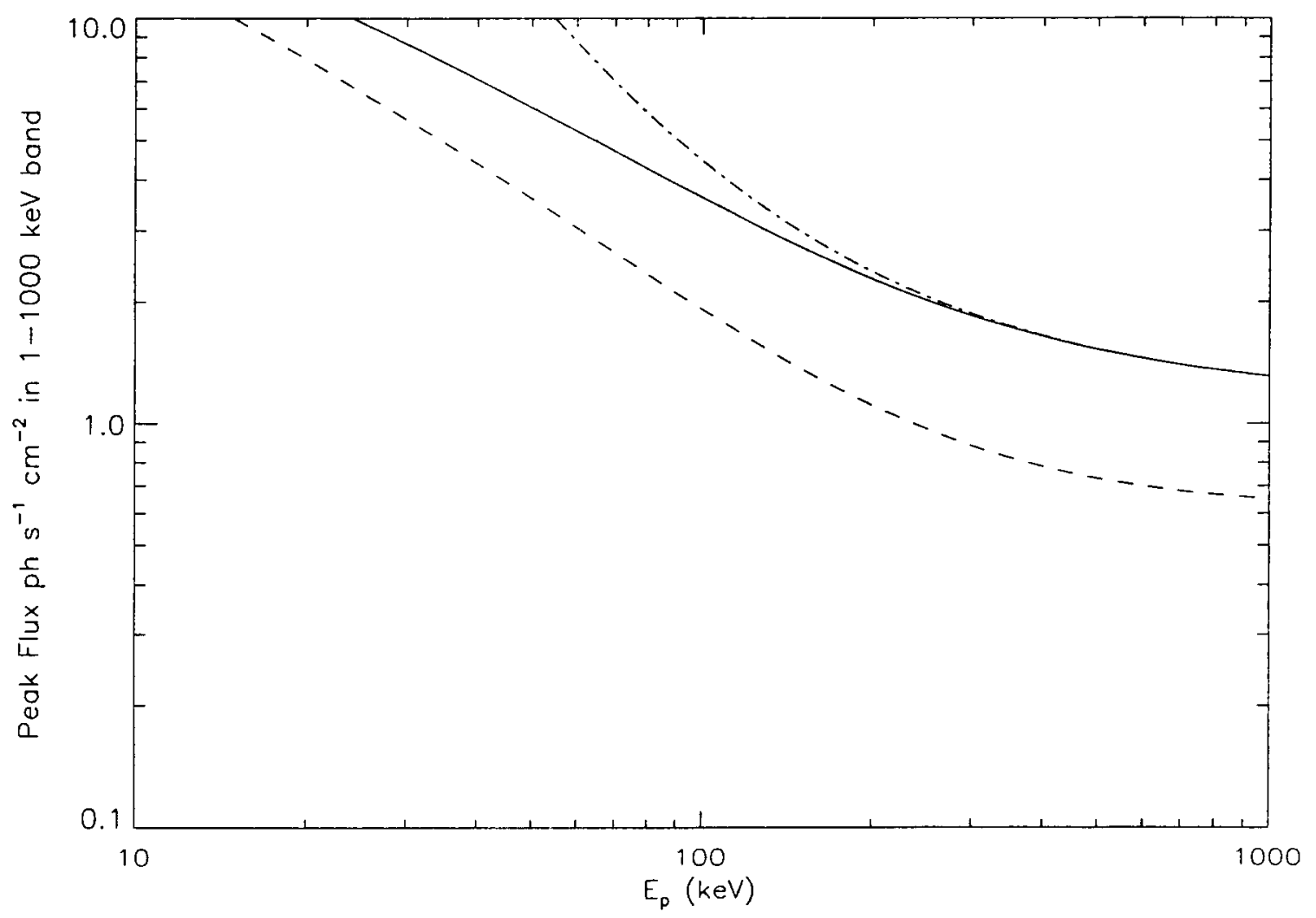

Fig. 4.- Peak flux (1-1000 keV) threshold of the BeppoSAX GRBM. The sensitivity for the on-board trigger is shown. Solid line- $\alpha=-1, \beta=-2$; dashed line $\alpha=-0.5, \beta=-2$; dot-dashed line- $\alpha=-1, \beta=-3$. 


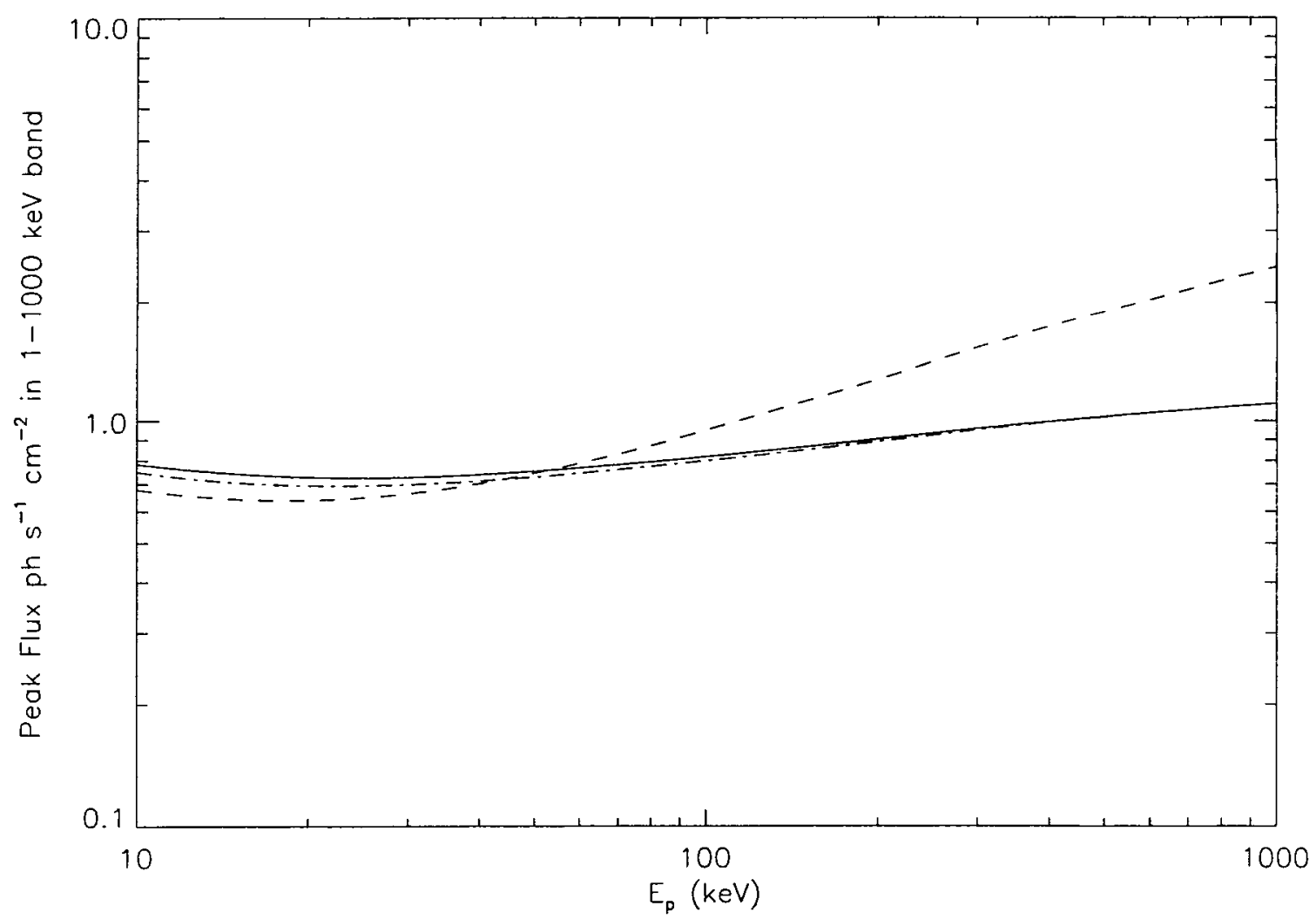

Fig. 3.- Peak flux (1-1000 keV) threshold of the BeppoSAX WFC. Solid line $-\alpha=-1$, $\beta=-2$; dashed line $\alpha=-0.5, \beta=-2$; dot-dashed line $-\alpha=-1, \beta=-3$. 


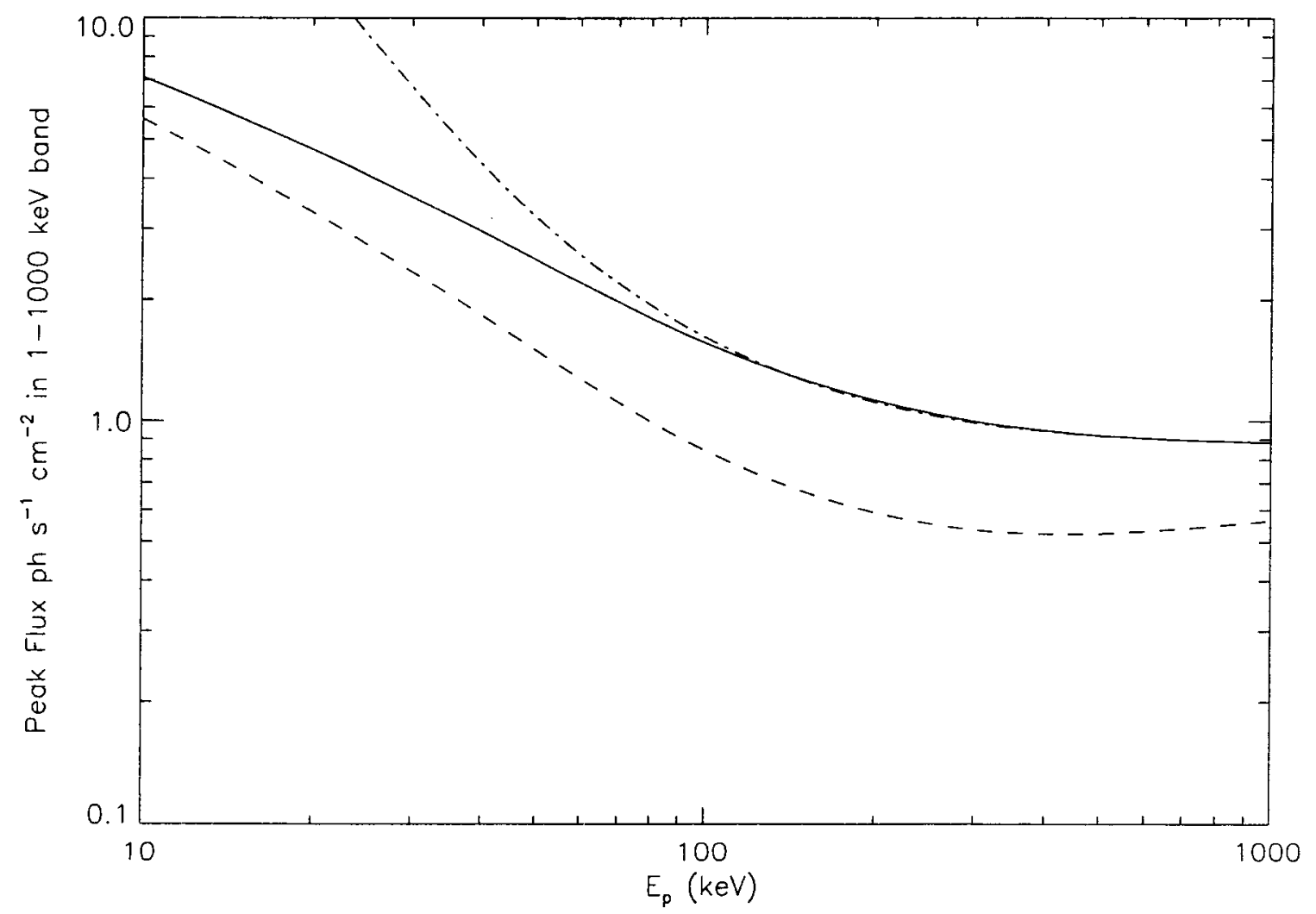

Fig. 2.- Peak flux (1-1000 keV) threshold of BATSE LAD detectors. Solid line- $\alpha=-1$, $\beta=-2$; dashed line- $\alpha=-0.5, \beta=-2$; dot-dashed line- $\alpha=-1, \beta=-3$. 


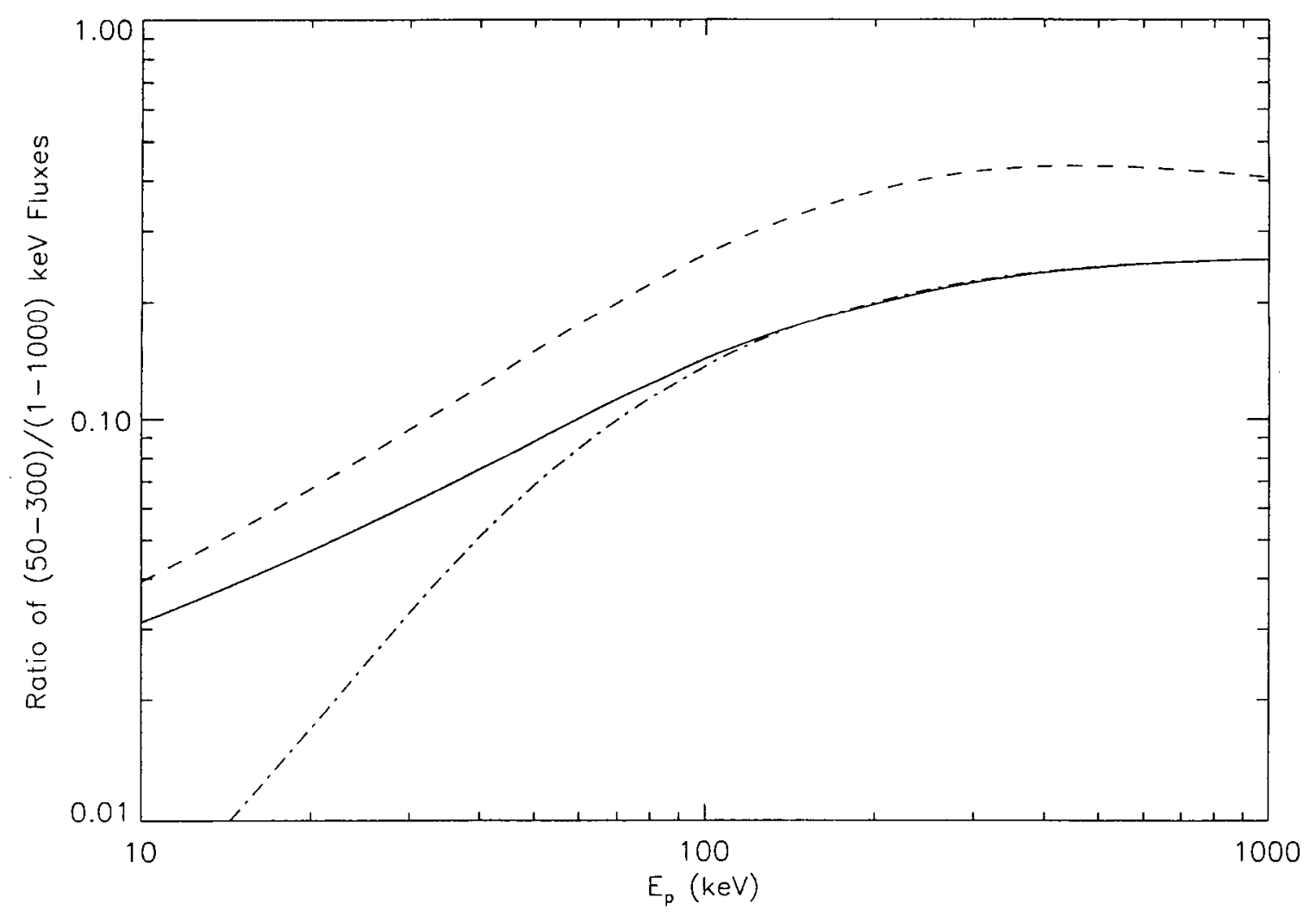

Fig. 1.- Ratio of fluxes in $50-300 \mathrm{keV}$ to $1-1000 \mathrm{keV}$ bands. Solid line $-\alpha=-1, \beta=-2$; dashed line $-\alpha=-0.5, \beta=-2$; dot-dashed line $-\alpha=-1, \beta=-3$. 
Table 1. Detector Parameters

\begin{tabular}{|c|c|c|c|c|c|c|c|c|}
\hline & BATSE $^{a}$ & $W F C^{b}$ & GRBM $^{\mathrm{b}}$ & $\mathrm{WXM}^{\mathrm{c}}$ & FREGATE $^{\mathrm{c}}$ & Swift & $\mathrm{GBM}^{\mathrm{d}}$ & $E X I S T$ \\
\hline$A^{\mathrm{e}}$ & 2025 . & 650. & 1136 & $213.6^{\mathrm{f}}$ & $39.6^{\mathrm{g}}$ & 7200 & 127 & $3000^{\mathrm{h}}$ \\
\hline$f_{\text {det }}^{i}$ & 1. & 0.8 & 1. & 0.938 & 1. & 0.72 & 1. & 1 \\
\hline$f_{\text {mask }}{ }^{\mathrm{j}}$ & 1. & 0.27 & 1. & 0.33 & 1. & 0.5 & 1. & 0.5 \\
\hline$\Omega^{\mathrm{k}}$ & $\pi$ & 0.123 & $\pi$ & 0.802 & 0.311 & 1.33 & $\pi$ & 0.704 \\
\hline$\sigma_{0}^{1}$ & $6.74(5.5)$ & 4 & $5.66(4)$ & 5.9 & 4.5 & 8 & 4.5 & 5 \\
\hline \multirow[t]{4}{*}{$\Delta E^{\mathrm{m}}$} & $50-300$ & $1.8-28$ & $40-700$ & $2-25$ & $6-40$ & $15-30$ & $50-300$ & $10-70$ \\
\hline & & & & & $6-80$ & $15-50$ & & $40-200$ \\
\hline & & & & & $32-400$ & $30-75$ & & $70-350$ \\
\hline & & & & & $>400$ & $50-150$ & & $100-1000$ \\
\hline
\end{tabular}

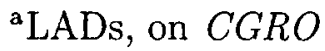

${ }^{\mathrm{b}} \mathrm{On} \operatorname{BeppoSAX}$

${ }^{\mathrm{c}} \mathrm{On} H E T E-2$

${ }^{\mathrm{d}} \mathrm{NaI}(\mathrm{Tl})$ detector, on $G L A S T$

${ }^{\mathrm{e}}$ Geometric detector area, in $\mathrm{cm}^{2}$

'Area of a single detector; sensitivity calculated for 2 detectors

${ }^{\mathrm{g}}$ Area of a single detector; sensitivity calculated for 4 detectors

${ }^{\text {h }}$ Area of a single module; sensitivity is calculated for a telescope of 9 modules. Note that EXIST will include 3 telescopes.

${ }^{\mathrm{i}}$ Fraction of detector plane that is active.

${ }^{\mathrm{j}}$ Fraction of the coded mask that is open.

${ }^{k}$ Average solid angle for the aperture flux

${ }^{1}$ Effective threshold significance, including the angle between the burst and the detector normal; the nominal significance is in parentheses

m Trigger energy band, in $\mathrm{keV}$; may change for current and future missions 
Strohmayer, T., Fenimore, E., Murakami, T., \& Yoshida, A. 1998, ApJ, 500, 873

Sullivan, J. D. 1971, NIM, 95, 5

von Kienlin, A., et al. 2000, in the 4th Integral Workshop, Alicante, 2000 


\section{REFERENCES}

Amati, L. 1999, thesis at Universitá "La Sapienza" di Roma (http://tonno.tesre.bo.cnr.it/ amati/tesi/tesi.html)

Atteia, J-L., et al. 2002, in Gamma-Ray Burst and Afterglow Astronomy 2001: A Workshop Celebrating the First Year of the HETE Mission, eds. R. Vanderspek and G. Ricker, in press [astro-ph/0202515]

Band, D. L. 2002, ApJ, 578, 806

Band, D. L., et al. 1993, ApJ, 413, 281

Feroci, M., et al. 1997, Proc. SPIE Conference (San Diego, 1997), 3114, 186 [astro$\mathrm{ph} / 9708168]$

Fishman, G. J., et al. 1989, in Proceedings of the Gamma Ray Observatory Science Workshop, 2-39

Guidorzi, C. 2001, thesis at the University of Ferrara (http://www.fe.infn.it/ guidorzi/doktorthese/)

Grindlay, J., et al. 2002, in Gamma-Ray Burst and Afterglow Astronomy 2001: A Workshop Celebrating the First Year of the HETE Mission, eds. R. Vanderspek and G. Ricker, in press

Gruber, D. E. 1992, in The X-ray Background. Collected Papers and Reviews from a Workshop held in Laredo, Spain, September, 1990, eds. X. Barcons \& A. C. Fabian, (Cambridge: Cambridge University Press), p. 44

Heise, J., in't Zand, J., Kippen, R. M., \& Woods, P. M. 2001, in Gamma-Ray Bursts in the Afterglow Era, 17-20 October, 2000, eds. E. Costa, F. Frontera, \& J. Hjorth (Berlin: Springer), p. 16

Jager, R., et al. 1997, A\&A Suppl, 125, 557

Kawai, N., et al. 2002, in Gamma-Ray Burst and Afterglow Astronomy 2001: A Workshop Celebrating the First Year of the HETE Mission, eds. R. Vanderspek and G. Ricker, in press

Mallozzi, R., et al. 1995, ApJ, 454, 597

Preece, R., et al. 2000, ApJS, 126, 19 
trigger on X-Ray Flashes (XRFs), transients with low $E_{p}$ (many of the XRFs Beppo-SAX's WFC detected are untriggered events in the BATSE data-Kippen et al. 2002), whereas the FREGATE has detected XRFs. Mallozzi et al. (1995) showed that on average bursts become softer as they become fainter. Kippen et al. show that the XRFs detected by Beppo-SAX appear to be the low intensity extension of the Mallozzi et al. trend.

A comparison of the BATSE and Swift sensitivities (Figures 2 and 7) indicates that on the $1 \mathrm{~s}$ timescale Swift may not detect fainter bursts with $E_{p}>100 \mathrm{keV}$, but it will detect soft bursts (e.g., XRFs) that are a factor of $\sim 2$ fainter than BATSE's threshold. Swift will trigger on timescales both shorter and longer than the $64 \mathrm{~ms}, 256 \mathrm{~ms}$ and $1.024 \mathrm{~s}$ timescales on which BATSE triggered, and thus will detect fainter bursts with certain types of lightcurves (e.g., short bursts).

\section{Summary}

I have presented a method of comparing the energy sensitivities of different gamma-ray burst detectors. Since the emphasis is on the energy sensitivity, I assume that all detectors trigger on a $\Delta t=1 \mathrm{~s}$ accumulation time; the sensitivity for different accumulation times can be estimated from an ensemble of burst lightcurves (Band 2002). I propose presenting the intensity of a burst in a common unit: the peak photon flux integrated over the $1-1000 \mathrm{keV}$ band. The threshold peak flux $F_{T}$ can then be calculated for each detector for a particular spectral shape. The peak energy $E_{p}$ (the energy of the maximum of $E^{2} N_{E} \propto \nu f_{\nu}$ ) is the first order measure of a spectrum's hardness. Thus a plot of $F_{T}$ vs. $E_{p}$ is a useful summary of a detector's sensitivity.

An application of this methodology shows that the sensitivity of BATSE and Swift are approximately equal above $E_{p} \sim 100 \mathrm{keV}$, while Swift will be more sensitive for bursts with lower values of $E_{p}$. As expected for smaller NaI detectors, the GBM-NaI system will be much less sensitive than BATSE was. FREGATE is surprisingly sensitive, largely because of the wide energy band it covers. Finally, with its large area, EXIST will be more sensitive than previous detectors.

I would like to thank L. Amati, M. Briggs, N. Gehrels, J. Grindlay, C. Guidorzi, J. Heise, J. Hong, N. Kawai, C. Markwardt, J. Norris and R. Preece for discussions and comments upon this work. 
and for different parts of the spacecraft's orbit.

Nonetheless, the curves are a measure of the relative sensitivities of the different detectors. The GBM's NaI detectors will be less sensitive than the BATSE LADs because they will have much less area. The FREGATE is somewhat more sensitive than the BATSE LADs because it usually triggers on a less significant rate increase ( $4.5 \sigma$ vs. $6.74 \sigma$ ); the FREGATE's smaller area is offset by the small FOV which reduces the aperture flux (see Table 1). Also, FREGATE triggers of a variety of $\Delta E$ while BATSE triggered on only one value of $\triangle E$. EXIST will be more sensitive than Swift because the aperture flux (per detector area) is almost a factor of 2 smaller while the detector area is larger. Note that although the total detector area of just one of EXIST's three telescopes will be $27,000 \mathrm{~cm}^{2}$, no point in the FOV is in the fully coded region of all 9 modules. These relative sensitivities are also affected by the trigger significance required of a rate increase.

The energy dependence of the detector efficiency affects the energy dependence of the sensitivity. CZT has high efficiency below $\sim 100 \mathrm{keV}$ and then decreases, while NaI's efficiency peaks at $\sim 100 \mathrm{keV}$ and remains high until $\sim 1 \mathrm{MeV}$. Thus the sensitivity of the BATSE LADs (which were NaI detectors) and the GBM NaI detectors decreases significantly for $E_{p}<100 \mathrm{keV}$, while the sensitivity of the CZT detectors (Swift and EXIST) decrease much less for low $E_{p}$. EXIST will use thicker CZT detectors than Swift, which will increase the high energy efficiency, and thus EXIST's high energy sensitivity is greater than Swift's by more than would be predicted by the increase in area.

A lower low energy cutoff also increases the low energy sensitivity since more of the spectrum can be detected. This explains the FREGATE's low energy sensitivity relative to Swift's, even though the FREGATE uses NaI and Swift will use CZT: FREGATE is sensitive above $6 \mathrm{keV}$ and Swift's spectrum will begin at $\sim 15 \mathrm{keV}$.

As expected, the trigger energy bands also affect the energy sensitivity. The cusps evident in the curves for FREGATE, Swift and EXIST result from high ( $\sim 50-150 \mathrm{keV})$ and low $(\sim 15-50 \mathrm{keV})$ trigger bands. Figure 10 shows the sensitivity of the four trigger energy bands proposed for Swift.

\subsection{Implications}

Gamma-ray bursts will populate the $E_{p^{-}}-F_{T}$ plane shown in Figures 2-9. Thus the detector sensitivities shown on these figures show which burst populations the detectors will detect. BATSE and FREGATE have comparable sensitivities above $E_{p}=100 \mathrm{keV}$ whereas the FREGATE is significantly more sensitive below $E_{p}=100 \mathrm{keV}$. Thus BATSE did not 
triggers where two or more detectors must trigger. The orientation of the 12 detectors has not been finalized, but the smallest angle between two detectors will be of order $\sim 30^{\circ}$, and thus the most sensitive points in the FOV will have an angle to the second most brightly illuminated detector of $\sim 15^{\circ}$. Since $\cos \left(15^{\circ}\right) \sim 1$ I use the threshold significance of $\sigma_{0}=4.5$. The trigger will use the standard BATSE energy band of 50-300 keV.

\subsection{EXIST}

The Energetic X-ray Imaging Survey Telescope (EXIST) is currently proposed to be a free-flying mission to detect gamma-ray bursts and conduct a hard X-ray sky survey (Grindlay et al. 2002). EXIST will carry three identical telescopes, each of which will consist of 9 coded mask modules. The modules' detector planes will be canted with respect to each other by $10-12.5^{\circ}$. A module's detector plane will be $3000 \mathrm{~cm}^{2}$ of $\mathrm{CZT}$ in front of a CsI anticoincidence shield. The CZT detectors will be 5-10 mm thick, increasing the high energy efficiency relative to Swift; I use the efficiency curve for $5 \mathrm{~mm}$ thick CZT in my calculations. Additional CsI planes will form $90 \mathrm{~cm}$ high collimator walls between the modules. The active CsI shielding can also be used as a high energy gamma-ray burst detector, but its effects will not be considered here. A curved coded mask will arch over all of a telescope's 9 modules $150 \mathrm{~cm}$ above the detector planes.

To determine a telescope's sensitivity I calculate the sensitivity for a single module, and then consider how the modules' sensitivity add together over the sky for a single telescope. The fully-coded regions of all 9 modules in a telescope do not overlap. The maximum sensitivity for the current design is 1.92 times the sensitivity of a single module.

\section{Discussion}

\subsection{Detector Comparison}

The maximum sensitivities for the different detectors are presented in Figures 2-9; the fraction of a detector's FOV at or near the maximum sensitivity varies from detector to detector. The sensitivity decreases away from the detector's normal because of the projection of the detector plane to the burst (proportional to the cosine of the inclination angle). In the partially coded region of a coded mask detector the detector's walls shadow part of the detector plane. In addition, the background induced by the particle flux, which is modelled crudely in my study, varies over an orbit, raising and lowering the sensitivity. Thus the curves in Figures 2-9 will shift up and down (mostly up) for different angles to the detector 
spread over $\sim 7200 \mathrm{~cm}^{2}$. A D-shaped coded mask with a total area of $3.2 \mathrm{~m}^{2}$ and $5 \times 5 \mathrm{~mm}^{2}$ cells will be one meter above the detector plane. I use an efficiency curve provided by C. Markwardt (personal communication, 2002).

My formula for the average solid angle $\Omega$ seen by the detector plane is for a rectangular mask above a rectangular detector plane; the BAT has a more complicated geometry. To calculate $\Omega$ I assume the mask is $120 \times 250 \mathrm{~cm}^{2}$ and the detector plane is $60 \times 120 \mathrm{~cm}^{2}$. Only a fraction $\sim 0.72$ of this detector plane is active, but the dimensions of the region over which the active area is spread are required. Note that $\Omega$ is used to calculate the background rate, and the threshold flux is proportional to the square root of the background; therefore the result will not be very sensitive to small errors in $\Omega$ resulting from this approximation.

The Swift rate trigger will be very flexible, utilizing many different energy bands, background estimates, and accumulation times (E. Fenimore, personal communication, 2002). These different triggers will use different significances. To compare the sensitivity as a function of energy of different detectors, I consider only $\Delta t=1 \mathrm{~s}$, and I use the energy bands currently planned (see Table 1; D. Palmer, personal communication, 2002). Although the rate triggers for $\Delta t=1 \mathrm{~s}$ may first trigger on $\sigma_{0} \sim 6$, the requirement that a new source appear in the image of the source image effectively raises $\sigma_{0}$ to $\sim 8$. Note that the BAT will trigger not only on the rate from the entire detector plane, but also on the rate from subsets, increasing the sensitivity in the partially-coded FOV (E. Fenimore, personal communication, 2002). The maximum sensitivity will be at the center of the fully-coded region normal to the BAT.

\subsection{GLAST's GBM}

The Gamma-ray Burst Monitor (GBM) planned for the Gamma-ray Large Area Space Telescope (GLAST) will consist of $12 \mathrm{NaI}$ detectors to cover the 5-1000 keV band and two $\mathrm{BGO}$ detectors to cover the 1-30 MeV band. The purpose of the GBM is to detect bursts in or near the FOV of the Large Area Telescope (LAT), GLAST's main instrument, and to characterize the bursts. Since the LAT is a high energy gamma-ray detector, the sensitivity to particularly hard bursts is relevant. Here I focus on the sensitivity of the NaI detectors, which will be built around flat $127 \mathrm{~cm}^{2} \mathrm{NaI}(\mathrm{Tl})$ crystals that will each view $\sim$ half the sky (von Kienlin et al. 2000). The two BGO detectors will provide spectral coverage between the NaI detectors and the LAT, but will not be useful for detecting bursts (as I have verified with this paper's methodology).

Included among the variety of burst triggers for the NaI detectors will be BATSE-like 
coded mask $18.7 \mathrm{~cm}$ above two position-sensitive proportional counters (PSPCs). The mask is $1 / 3$ open. The geometric area of each PSPC is $8.35 \times 12 . \mathrm{cm}^{2}$, and they are separated by a gap of $1 \mathrm{~cm}$. The detectors operate over the $2-25 \mathrm{keV}$ band. I use the background count rate of $\sim 700$ for both detectors provided by Kawai et al. (2002). A variety of triggers are used, with different trigger significances; I use $\sigma_{0}=5.9$ for the $\Delta t=1 \mathrm{~s}$ accumulation in the rate summed over both detectors. A burst must be imaged after a rate trigger, effectively raising the significance for the detection of a burst, but I do not attempt to model this effect.

\subsection{HETE-2's FREGATE}

The FREGATE (Atteia et al. 2002) is a set of $4 \mathrm{NaI}(\mathrm{Tl})$ detectors on the High Energy Transient Explorer II (HETE-2). The FREGATE's goals are a) the detection of bursts for localization by the imaging cameras, b) burst spectroscopy and c) monitoring hard X-ray transient sources. Each detector has a circular area of $39.6 \mathrm{~cm}^{2}$. The active area is exposed to the sky without a coded mask, and the FREGATE has no localization capabilities. The shield around each $\mathrm{NaI}$ crystal extends above the front surface of the crystal, reducing both the FOV and the aperture flux. The detectors are sensitive in the 6-400 keV band, and triggers in the $6-40 \mathrm{keV}, 6-80 \mathrm{keV}, 32-400 \mathrm{keV}$, and $>400 \mathrm{keV}$. The trigger uses a significance between $\sigma_{0}=4.5$ and 6 . For the maximum sensitivity I assume that $\sigma_{0}=4.5$ for a burst on the detector normal, and that the signals from all four detectors are summed. Note that false triggers are eliminated by requiring a rate increase in non-overlapping pairs of detectors. To calculate the average solid angle of the sky $\Omega$ I approximate the detectors as squares.

\subsection{Swift's BAT}

The Burst Alert Telescope (BAT) is Swift's gamma-ray instrument. The BAT will detect the gamma-ray burst, localize it, and instruct the spacecraft to slew so that the burst location is in the much smaller FOV of the X-ray and optical telescopes coaligned with the BAT. The BAT will use a two step trigger: first, it will detect an increase in the count rate, and second, it will image the burst. Only if the count rate increase originates from a point source will the event be considered an astrophysical transient. Here I consider only the count rate trigger.

The BAT will consist of a rectangular detector plane of $32,768 \mathrm{CZT}$ detectors, each $4 \times 4 \mathrm{~mm}^{2}$; because of the packaging of the detectors, the total active area of $5243 \mathrm{~cm}^{2}$ is 
was triangular; I present the sensitivities at the center.

The WFC did not trigger on-board, but instead the rates in $1 \mathrm{~s}$ and $8 \mathrm{~s}$ time bins were analyzed on the ground. Subsequently the rates accumulated over 1, 5 and 20 minutes were also searched for transients. A $\sigma_{0}=4$ increase in the count rate from one WFC unit triggered further analysis: the set of time bins with the highest signal-to-noise ratio were used to create an image, and a point source with a $5.5 \sigma$ significance was required to consider the burst real (J. Heise, personal communication, 2002). Thus for the analysis here $\sigma_{0}=4$.

\subsection{BeppoSAX's GRBM}

The Gamma-Ray Burst Monitor on BeppoSAX consisted of the four $1136 \mathrm{~cm}^{2}$ area, $1 \mathrm{~cm}$ thick CsI(Na) shields around the Phoswich Detection System (Feroci et al. 1997; Amati 1999); detecting bursts was the secondary role of these shields. For most of the mission the system triggered when the $40-700 \mathrm{keV}$ rate in two detectors accumulated over $\Delta t=1 \mathrm{~s}$ increased by more than $\sigma_{0}=4$ (Feroci et al. 1997; Amati 1999).

The average background count rate in the trigger band for each detector was $\sim 1000 \mathrm{ct}-$ $\mathrm{s} / \mathrm{s}$, and I use this rate rather than model the background count rate. The effective areas of the four detectors differed because of material that was in front of them, but I model them as having been identical. Because the count rate must have increased by $\sigma_{0}=4$ in two detectors, the most sensitive point in the GRBM's FOV for the on-board trigger was between the normal to two adjoining detectors, that is, at an angle of $45^{\circ}$ from each detector normal. Thus the effective significance was $\sigma_{0}=4 \sqrt{2}=5.66$.

The ultimate GRBM burst database is the result of a search on the ground with a variety of trigger criteria (Guidorzi 2001) utilizing the rates in the $40-700 \mathrm{keV}$ and the $>100 \mathrm{keV}$ bands from different sets of detectors. The background was estimated either as a constant rate calculated from count rates before the burst or as a linear fit to count rates before and after the burst. These complicated trigger criteria might lower the sensitivity curve by $\sim 1 / 3$. However, in the figure I show only the sensitivity for the on-board trigger.

\subsection{HETE-2's WXM}

The Wide-field X-ray Monitor (WXM) is the primary detector on HETE-2 for the localization of gamma-ray bursts (Kawai et al. 2002). The WXM consists of two coded mask $\mathrm{X}$-ray detector units sensitive to the burst's position in one dimension; the units' orientations are orthogonal to each other, providing a two-dimensional position. Each WXM unit has a 
this study is to compare detectors with different energy responses and triggering on different energy bands, and not to study the effect of different trigger times. Almost all detectors include $\Delta t=1 \mathrm{~s}$ among their set of trigger times, and this will be the value used here.

In the next section I present the detectors in this study; Table 1 summarizes some of the detector parameters. I use a detector's maximum sensitivity, even if achieved over only a small region within the field-of-view (FOV).

\section{The Detectors}

\subsection{CGRO's BATSE}

BATSE consisted of eight modules, each with two types of detectors: the Large Area Detector (LAD) for burst detection, localization and monitoring; and the Spectroscopy Detector (SD) for spectral analysis. Thus the LADs are relevant to this study. The LADs were built around large $\left(2025 \mathrm{~cm}^{2}\right)$, flat $\mathrm{NaI}(\mathrm{Tl})$ crystals. The LADs in the eight modules were parallel to the faces of a regular octahedron. The LAD effective area curve is found in Fishman et al. (1989). The LADs operated in the $20-2000 \mathrm{keV}$ band, but usually triggered in the 50-300 keV band.

BATSE triggered as a whole when two or more LADs each triggered on an increase greater than $\sigma_{0}=5.5$. Therefore the sensitivity depended on the second most brightly illuminated detector for which the cosine of the angle to the source (the factor by which the flux is diminished) varied between $1 / 3$ (when the burst was along the normal to the most brightly illuminated detector) and 0.8165 (when the burst was exactly between two detectors). Note that this analysis ignores the effects of scattering off of the spacecraft and the Earth's atmosphere. In this study I use the maximum sensitivity in the detector's FOV. Thus for BATSE the required significance is equivalent to $\sigma_{0}=5.5 / 0.8165=6.74$.

\subsection{BeppoSAX's WFC}

BeppoSAX's Wide Field Cameras (WFCs) were two anti-parallel coded mask detectors which pointed perpendicular to the axis of the Narrow Field Instruments (Jager et al. 1997). The detector plane was a $25.5 \times 25.5 \mathrm{~cm}^{2}$ multi-wire proportional counter that was active over 0.8 of its area. Only $1 / 3$ of the $1 \mathrm{~mm}^{2}$ mask pixels were open, but because of the supports for the mask pixels, the actual open area of an open pixel was $0.9 \times 0.9 \mathrm{~mm}^{2}$. Because the mask and the detector plane were almost exactly the same dimension, the spatial sensitivity 
rate increase is assumed to result from the burst flux. In most cases I model the background (counts $\mathrm{s}^{-1} \mathrm{keV}^{-1} \mathrm{~cm}^{-2}$ ) as

$$
B(E)=\epsilon(E) \Omega f_{\text {mask }} N_{B}(E)+B_{\text {int }}
$$

where $N_{B}(E)$ is the diffuse high energy background (Gruber 1992), $\Omega$ is the average solid angle of the sky as seen from the detector plane (calculated from the corrected formulae in Sullivan 1971), $f_{\text {mask }}$ is the fraction of the coded mask that is open, and $B_{\text {int }}$ is the internal background. Note that the aperture flux resulting from the diffuse background is detected with the detector's efficiency $\epsilon(E)$. This background model is clearly a simplification because it ignores the background induced by the particle flux, scattering off the spacecraft and the Earth's atmosphere, and activation of the detector and its environs. At higher energies (e.g., $\sim 100 \mathrm{keV}$ ) the instrument walls may become transparent, and $\Omega$ may increase; this effect is not considered here. Nonetheless, this model gives an approximate magnitude and energy dependence. In some cases I use the observed background rates.

The trigger has a preset threshold significance

$$
\sigma_{0}=\frac{A f_{\text {det }} f_{\text {mask }} \Delta t \int_{E_{1}}^{E_{2}} \epsilon(E) N_{T}(E) d E}{\sqrt{A f_{\text {det }} \Delta t \int_{E_{1}}^{E_{2}} B(E) d E}}
$$

where $N_{T}(E)$ is the peak burst flux at the threshold, and $f_{\text {det }}$ is the fraction of the detector plane that is active. In eq. 4 the numerator is the number of counts from the burst and the denominator is the square root of the number of counts expected from the background rate. Therefore, the broadband photon flux at the detector's threshold is

$$
F_{T}=\frac{\int_{E_{l}}^{E_{h}} N_{T}(E) d E}{\int_{E_{1}}^{E_{2}} \epsilon(E) N_{T}(E) d E} \frac{\sigma_{0} \sqrt{\int_{E_{1}}^{E_{2}} B(E) d E}}{f_{\text {mask }} \sqrt{A f_{\operatorname{det}} \Delta t}} .
$$

Note that the ratio of integrals over $N_{T}$ eliminates the unknown normalization, and results in the inverse of the average efficiency. I vary the spectral shape of $N_{T}(E)$ by varying $E_{p}$, holding $\alpha$ and $\beta$ fixed. The resulting $F_{T}\left(E_{p}\right)$ compares different detectors.

For each detector I need information about the detector and about the burst trigger. For the detector I need the area $A$, the detector efficiency $\epsilon(E)$, the fraction of the detector that is active $f_{\text {det }}$, the fraction of the coded mask that is open $f_{\text {mask }}$, the average solid angle $\Omega$, and the internal background $B_{\text {int }}$; for the trigger I need the effective threshold significance $\sigma_{0}$ and the trigger energy band $E_{1}-E_{2}$. Most detector papers present a plot of $\epsilon(E)$; I model $\epsilon(E)$ as a series of power laws between representative values of $E$. Detectors use triggers with a variety of trigger times $\Delta t$ and energy ranges (defined by $E_{1}$ and $E_{2}$ ). The goal of 
detectors. In $\S 2$ I discuss the methodology used in this comparison, while $\S 3$ presents the relevant information about each detector. The results and their implications are discussed in $\S 4$.

\section{Methodology}

I assume that the burst spectrum can be described by the "GRB" function (Band et al. 1993),

$$
\begin{array}{ccl}
N(E)= & N_{0}\left(\frac{E}{100 \mathrm{keV}}\right)^{\alpha} \exp \left[-\frac{E}{E_{0}}\right] & ; E \leq E_{b} \\
& N_{0}\left(\frac{E_{b}}{100 \mathrm{keV}}\right)^{\alpha-\beta} \exp [\beta-\alpha]\left(\frac{E}{100 \mathrm{keV}}\right)^{\beta} ; \quad E>E_{b} \\
\text { where } & E_{b}=(\alpha-\beta) E_{0} & \text { and } E_{p}=(\alpha+2) E_{0} .
\end{array}
$$

$N(E)$ has units of photons $\mathrm{s}^{-1} \mathrm{keV}^{-1} \mathrm{~cm}^{-2}$. I compare the sensitivity of different detectors by holding $\alpha$ and $\beta$ fixed, and varying $E_{p}$. To compare spectra with different values of $E_{p}$ I use the integral of $N(E)$ over a broad energy band

$$
F=\int_{E_{l}}^{E_{h}} N(E) d E
$$

where $E_{l}=1 \mathrm{keV}$ and $E_{h}=1000 \mathrm{keV}$. Fig. 1 compares the fluxes in the $50-300 \mathrm{keV}$ and 1-1000 keV bands as a function of $E_{p}$ for different sets of $\alpha$ and $\beta$. As can be seen, the 1-1000 keV flux includes more photons for small $E_{p}$.

The sensitivity of a detector to a burst with a particular spectral shape depends on its burst trigger and hardware properties such as the detector area $A$, the fraction of the detector that is active $f_{\text {det }}$, the fraction of the coded mask that is open $f_{\text {mask }}$, and the efficiency $\epsilon(E)$. If the detector does not have a coded mask, then $f_{\text {mask }}=1$. Most gamma-ray detectors do not have a one-to-one mapping between a photon's energy and the energy channel the detector assigns a detected count. If the incoming photon spectrum is binned in energy, the relationship between the photon energy bins and the detector's energy channels is a detector response matrix (DRM) with off diagonal elements and not a vector of the detection efficiency for each energy bin. However, since I calculate the count rates over broad energy bands, the DRM can be approximated by an efficiency vector.

The typical burst trigger looks for a statistically significant increase in the detector's count rate above the background in the energy band between $E_{1}$ and $E_{2}$ in a time bin $\Delta t$. The significance of the increase is measured in units of the expected fluctuation scale of the background, i.e., the square root of the expected number of background counts. The count 